\title{
Intervenções Comportamentais no Ensino de Atenção Conjunta para Crianças com Autismo: Uma revisão de literatura
}

\author{
Flávia Teresa Neves Silva Bacelar* \\ Carlos Barbosa Alves de Souza \\ Universidade Federal do Pará, Belém, Brasil
}

\begin{abstract}
RESUMO
Este trabalho revisou estudos baseados na metodologia e procedimentos da Análise do Comportamento que investigaram o ensino da atenção conjunta (AC) para crianças com autismo, procurando descrever e analisar as variáveis envolvidas no ensino de resposta de atenção conjunta (RAC) e de iniciação de atenção conjunta (IAC). Foram avaliados: componentes da AC e classes de respostas de RAC e IAC treinados; procedimentos utilizados; estímulos discriminativos e reforçadores usados; participação de familiares; e resultados de aquisição e generalização. Os resultados da revisão mostraram o sucesso em estabelecer RAC e IAC através de procedimentos comportamentais e apontam quais estratégias podem favorecer a manutenção e generalização desses repertórios. Sugere-se a necessidade de estudos que avaliem a relação entre $\mathrm{AC}$ e operantes verbais básicos.
\end{abstract}

Palavras-chave: atenção conjunta; autismo; procedimentos comportamentais.

\section{ABSTRACT \\ Behavioral interventions for teaching joint attention to children with autism: A literature review}

This paper reviewed studies based on Behavior Analysis methodologies and procedures that investigated the teaching of joint attention (JA) for children with autism, trying to describe and analyze the variables involved in teaching joint attention responses (JAR) and initiation of joint attention (IJA). The following aspects were evaluated: JA components and responses classes for JAR and IJA trained; procedures used; discriminative stimuli and reinforcers used; family participation, and; results for acquisition and generalization. The results of the review showed the success in establishing RJA and IJA through behavioral procedures and indicated some strategies that may favor the maintenance and generalization of these repertoires. Futures studies should be carried out to investigate the relationship between JA acquisition and basic verbal operants.

Keywords: joint attention; autism; behavioral procedures.

O autismo é considerado um transtorno do desenvolvimento, caracterizado por déficits e excessos comportamentais que afetam a interação social e a comunicação (DSM 5 - American Psychiatric Association, 2013). Pessoas com autismo geralmente apresentam déficits de atenção conjunta (AC), o que é apontado como uma das causas de suas dificuldades linguísticas (Charman, 2003; Tager-Flusberg, Paul, \& Lord, 2005; Tsatsanis, 2005; Whalen, Schreibman, \& Ingersoll 2006).

A AC tem sido caracterizada na perspectiva da psicologia do desenvolvimento como a capacidade de compartilhar com o outro um evento ou objeto de interesse presente no ambiente (Morales et al., 2000; Mundy et al., 2007; Mundy \& Newell, 2007; White et al., 2011). Tem sido proposto que a habilidade de $\mathrm{AC}$ é composta por respostas de atenção conjunta (RAC) e iniciação de atenção conjunta (IAC). A RAC se refere à resposta da criança de seguir a direção para onde aponta ou olha outra pessoa. A IAC se refere aos comportamentos de contato visual, apontar ou demonstração que a criança emite de forma intercalada de uma pessoa para um objeto ou evento (ou vice-versa) (Mundy et al., 2007; Mundy et al., 2003; Mundy \& Newell, 2007). De maneira geral, tem sido apontada uma maior facilidade em ensinar RAC do que IAC 
para pessoas com autismo (Meindl \& CannellaMalone, 2011; White et al., 2011).

Na última década vem se desenvolvendo uma caracterização analítico-comportamental da $\mathrm{AC}$ em termos das relações funcionais estabelecidas entre situações discriminativas, comportamentos e suas consequências (Dube, MacDonald, Mansfield, Holcomb, \& Ahearn, 2004; Holth, 2005; Klein, MacDonald, Vaillancourt, Ahearn, \& Dube, 2009; MacDonald et al., 2006). Por exemplo, Dube et al. (2004) sugeriram que IAC e RAC são repertórios funcionalmente diferentes no processo inicial de aquisição. Na RAC a interação é iniciada pelo adulto que emite um mando ${ }^{1}$ (Skinner, 1957/1992) ao apontar ou olhar para um objeto/evento (ou também combinando essas respostas com vocalizações), e a resposta de atenção da criança ao estímulo é caracterizada como uma obediência instrucional (ver Greer \& Ross, 2008) mantida por reforçadores generalizados. Na IAC, o antecedente é uma operação motivadora $(\mathrm{OM})^{2}$ (e.g. presença de um adulto familiar e estímulos com propriedades discriminativas ressaltadas ou incomuns) que evoca a interação da criança com o adulto através da resposta de alternar o olhar entre o objeto/evento e $\mathrm{o}$ adulto (gaze shift), mantida por reforçadores sociais. Inicialmente a resposta de alternar o olhar entre o adulto e o objeto/evento se caracteriza como uma resposta de observação, que pode produzir uma consequência específica (ex. acesso a um brinquedo). Na medida em que se estabelece uma história em que a atenção do adulto tem a função de alterar a efetividade dos eventos reforçadores (potencializando ou reduzindo a aversividade deles), a resposta da criança de monitoramento da atenção do adulto passa a ser um comportamento verbal com função de mando por reforçadores condicionados socialmente mediados (Dube et al., 2004). Considerando isto, estímulos discriminativos, reforçadores sociais, bem como operações motivadoras podem ser arranjados para tornar RACs e/ou IACs mais prováveis.

A abordagem analítico-comportamental da AC tem produzido estudos experimentais que procuraram ensinar os repertórios de RAC e/ou IAC para crianças com autismo. Estes estudos utilizaram diversos procedimentos para ensinar classes de respostas de RAC e/ou IAC e apresentaram variações quanto aos resultados de aquisição e generalização (e.g. Krstovska-Guerrero \& Jones, 2013; Taylor \& Hoch, 2008; Whalen \& Schreibam, 2003). No entanto, não existe até o momento ne- nhuma revisão sistemática dessa literatura que permita uma análise mais precisa das variáveis envolvidas no ensino de AC para pessoas com autismo, desde uma perspectiva analítico-comportamental.

Este trabalho revisou estudos baseados na metodologia e procedimentos da Análise do Comportamento que investigaram o ensino da AC para crianças com autismo, procurando descrever $\mathrm{e}$ analisar as variáveis envolvidas no ensino dos componentes da AC. Foram avaliados os seguintes aspectos: A. Componente da AC treinado (RAC e/ou IAC); B1. Classes de respostas de RAC treinadas; B2. Classes de respostas de IAC treinadas; C. procedimentos específicos utilizados (e.g. tentativa discreta; treino de resposta pivotal); D. Estímulos discriminativos e reforçadores empregados; E. Participação de familiares no procedimento; e F. Resultados de aquisição e generalização.

\section{MÉTODO}

\section{Procedimento de busca}

Foi realizada uma busca sistemática nas bases de dados PubMed Central e Education Resources Information Center (ERIC), sem restrição de período, utilizando as palavras-chave "joint attention", "autism" e "training" como termos livres. Esse procedimento resultou em 324 artigos.

\section{Critérios de seleção dos artigos para análise}

A partir da leitura dos títulos e/ou resumos de cada artigo localizado, foram selecionados para análise aqueles que cumpriam os seguintes critérios: 1 . ser um relato de pesquisa publicado em um revista com revisão por pares; 2 . treinar pelo menos um dos componentes da AC; 3 . ter como participantes crianças diagnosticadas com autismo; 4. utilizar procedimentos da Análise do Comportamento (e.g. dicas; reforçamento); e 5. exibir controle experimental (i.e. demonstrar o efeito do treino no repertório do participante). Foram excluídos da análise os artigos que não preenchiam os cinco critérios listados acima.

\section{RESULTADOS}

Dezoito artigos preencheram os critérios de inclusão para análise. A Tabela 1 descreve os estudos considerando o perfil dos participantes, o delineamento experimental, e as variáveis selecionadas para análise no presente estudo: o componente da 
$\mathrm{AC}$ e as classes de respostas de RAC e IAC treinados, os procedimentos específicos utilizados, os tipos de estímulos discriminativos e reforçadores empregados, se houve participação de familiares no procedimento, e os resultados de aquisição e generalização. Na continuação, descrevemos os resultados da análise de cada uma dessas variáveis nos artigos selecionados.

A. Componente de AC treinado: dos 18 estudos analisados, nove ensinaram diretamente apenas um dos repertórios: quatro ensinaram RAC (Klein et al., 2009; Krstovska-Guerrero \& Jones, 2013; Martins \& Harris, 2006; Rocha, Schreibman, \& Stahmer, 2007) e cinco IAC (Jones, 2009; Kaale, Smith, \& Spoheim, 2012; MacDuff, Ledo, McClannahan, \& Krantz, 2007; Naoi, Tsuchiya, Yamamoto, \& Nakamura, 2008; Pollard, Betz, \& Higbee, 2012). Os nove estudos que buscaram ensinar ambos os repertórios apresentaram resultados díspares: aprendizagem apenas de RAC (Kasari, Gulsrud, Wong, Kwon, \& Locke, 2010; Taylor \& Hoch, 2008; Whalen \& Schreibman, 2003; Warreyn \& Royers, 2013; Wong, 2013) ou de ambos os repertórios (Gulsrud, Kasari, Freeman, \& Paparella, 2007; Isaksen \& Holth, 2009; Jones, Carr, \&
Feeley, 2006; Kasari, Freeman, \& Paparella, 2006).

B1. Classes de Respostas de RAC: as classes de respostas de RAC que foram treinadas mais frequentemente nos 18 estudos foram 'olhar para o objeto apontado por E.' (ex. Gulsrud et al., 2007; Isaksen \& Holth, 2009; Jones et al., 2006; Kasari et al., 2006, 2010; Rocha et al., 2007; Taylor \& Hoch, 2008; Warreyen \& Royers, 2013; Whalen \& Schreibman, 2003; Wong, 2013) e a 'discriminação da mudança no foco do olhar do adulto' (ex. Klein et al., 2009; Martins \& Harris, 2006; Rocha et al., 2007; Warreyen \& Royers, 2013; Whalen \& Schreibman, 2003; Wong 2013). Em Whalen e Schreibman (2003) e Isaksen e Holth (2009) a RAC foi instalada através de seis passos iniciados pelo experimentador, que progrediam quanto ao nível de dificuldade da resposta: 1. olhar para um objeto colocado na mão da criança; 2. olhar para um objeto balançado pelo experimentador; 3 . olhar para um objeto apresentado pelo experimentador; 4. olhar para o rosto do experimentador; 5. olhar em direção ao que era apontado pelo experimentador; e 6. seguir a direção do olhar do experimentador. 
Tabela 1

Participantes, delineamento e as variáveis analisadas nos artigos revisados no presente estudo (legenda das siglas no final da tabela)

\begin{tabular}{|c|c|c|c|c|c|c|c|}
\hline Autores & $\begin{array}{c}\text { Perfil dos } \\
\text { participantes }\end{array}$ & $\begin{array}{l}\text { Delineamento } \\
\text { experimental }\end{array}$ & $\begin{array}{l}\text { Componente ACl } \\
\text { Classes de Resp. }\end{array}$ & Intervenção & Estímulos & $\begin{array}{l}\text { Partic. de } \\
\text { familiares }\end{array}$ & $\begin{array}{l}\text { Aquisição e } \\
\text { Generalização }\end{array}$ \\
\hline $\begin{array}{l}\text { Whalen e } \\
\text { Schreibman } \\
\text { (2003) }\end{array}$ & $\begin{array}{l}5 \text { crianças com } \\
\text { autismo e } 6 \text { com } \\
\text { desenvolvi-mento } \\
\text { típico }\end{array}$ & $\begin{array}{l}\text { Linha de base múltipla } \\
\text { entre participantes }\end{array}$ & $\begin{array}{l}\text { RAC: a criança tinha que } \\
\text { olhar para objetos coloca- } \\
\text { dos na sua mão; olhar para } \\
\text { objetos apresentados por } \\
\text { E; OA; DMO } \\
\text { IAC: TO e A }\end{array}$ & $\begin{array}{l}\text { TTD para RAC e TRP para IAC; } \\
\text { Reforçamento positivo e punição } \\
\text { negativa; Hierarquia de DF, DG e } \\
\text { DV; RT; SG } 3 \text { meses após o treino }\end{array}$ & $\begin{array}{l}\text { SD: } B Q \text { menos preferidos e } \\
\text { painéis com figuras. } \\
\text { SRef: Consequências naturais } \\
\text { e tangíveis }\end{array}$ & Não & $\begin{array}{l}\text { Aquisição: Positiva } \\
\text { Generalização: } \\
\text { Negativa para IAC }\end{array}$ \\
\hline $\begin{array}{l}\text { Jones, Carr e } \\
\text { Feeley (2006) }\end{array}$ & $\begin{array}{l}\text { Estudo 1: } 5 \text { crianças } \\
\text { com autismo, de } 2 \text { e } \\
3 \text { anos; Estudo } 2 \text { e } \\
\text { 3: } 2 \text { crianças. Pre- } \\
\text { sença dos repertó- } \\
\text { rios: contato visual e } \\
\text { atender ao nome. }\end{array}$ & $\begin{array}{l}\text { Linha de base múltipla } \\
\text { entre as respostas }\end{array}$ & $\begin{array}{l}\text { RAC: AO } \\
\text { IAC: TO, A }\end{array}$ & $\begin{array}{l}\text { TTD para RAC e TI para IAC; } \\
\text { Hierarquia de DF e DV; RT; Mode- } \\
\text { lação; MES; Treino de generaliza- } \\
\text { ção e manutenção. Estudo 1: } \\
\text { Treino de Professores; Estudo 2: } \\
\text { Treino de pais; Estudo 3: verifica- } \\
\text { ção de mudanças colaterais e } \\
\text { validade social }\end{array}$ & $\begin{array}{l}\text { SD: BQ preferidos e de ativa- } \\
\text { ção, painéis com figuras e } \\
\text { atividades de rotina SRef: } \\
\text { consequências naturais, sociais } \\
\text { e comestíveis. }\end{array}$ & Sim & $\begin{array}{l}\text { Aquisição e Gene- } \\
\text { ralização: } \\
\text { Positivas para RAC } \\
\text { e IAC }\end{array}$ \\
\hline $\begin{array}{l}\text { Kasari et al. } \\
(2006)\end{array}$ & $\begin{array}{l}58 \text { crianças com } \\
\text { autismo, entre } 2-4 \\
\text { anos }\end{array}$ & $\begin{array}{l}\text { Delineamento de } \\
\text { grupo randomizado }\end{array}$ & $\begin{array}{l}\text { RAC: OA } \\
\text { IAC: TO, A, M, EO }\end{array}$ & $\begin{array}{l}\text { Treino da AC (TTD para RAC e TI } \\
\text { para IAC); Treino de BS; e Grupo } \\
\text { controle; Hierarquia de DF e DV; } \\
\text { Feedback corretivo; }\end{array}$ & $\begin{array}{l}\text { SD: BQ mecânicos e intera- } \\
\text { ções fisicas com E; } \\
\text { SRef: consequências sociais, } \\
\text { naturais, acesso ao SD. }\end{array}$ & Sim & $\begin{array}{l}\text { Aquisição e Gene- } \\
\text { ralização: } \\
\text { Positivas para RAC } \\
\text { e IAC }\end{array}$ \\
\hline $\begin{array}{l}\text { Martins e } \\
\text { Harris (2006) }\end{array}$ & $\begin{array}{l}3 \text { crianças com } \\
\text { autismo, com idade } \\
\text { de } 3 \text { e } 4 \text { anos. } \\
\text { Presença de reper- } \\
\text { tórios de IV, mando } \\
\text { e intraverbal entre } \\
\text { os participantes. }\end{array}$ & $\begin{array}{l}\text { Linha de base múltipla } \\
\text { com reversão entre } \\
\text { participantes }\end{array}$ & RAC:DMO & $\begin{array}{l}\text { TTD para RAC; Sequência treino: } \\
\text { 1. CN, GC, MF, A e tocar objeto; } 2 \text {. } \\
\text { CN, GC, MF, A sem tocar objeto; } 3 \text {. } \\
\text { CN, GC, MF e C; } 4 \text {. CN, GC e MF; } \\
\text { Uso dica física com e sem atraso; } \\
\text { RT; SG após treino. Sondas de } \\
\text { avaliação de IAC pela criança (A e } \\
\text { TO) }\end{array}$ & $\begin{array}{l}\text { SD: BQ e objetos preferidos } \\
\text { SRef: sociais, comestiveis e } \\
\text { consequências naturais. TRC }\end{array}$ & Não & $\begin{array}{l}\text { Aquisição e Gene- } \\
\text { ralização: Positivas } \\
\text { para RAC, negati- } \\
\text { vas para IAC }\end{array}$ \\
\hline $\begin{array}{l}\text { Gulsrud et al., } \\
\text { (2007) }\end{array}$ & $\begin{array}{l}35 \text { crianças com } \\
\text { autismo, entre } 2 \text { - } 4 \\
\text { anos }\end{array}$ & $\begin{array}{l}\text { Delineamento de } \\
\text { grupo }\end{array}$ & $\begin{array}{l}\text { RAC: OA; } \\
\text { IAC: A, M, TO }\end{array}$ & Ver Kasari et. al. (2006) & $\begin{array}{l}\text { SD: BQ luminosos e musicais. } \\
\text { SRef: Ver Kasari et. al. (2006) }\end{array}$ & Sim & $\begin{array}{l}\text { Aquisição e Gene- } \\
\text { ralização: } \\
\text { Positivas para RAC } \\
\text { e IAC }\end{array}$ \\
\hline $\begin{array}{l}\text { Rocha, } \\
\text { Schreibman e } \\
\text { Stahmer (2007) }\end{array}$ & $\begin{array}{l}3 \text { crianças com } \\
\text { autismo, entre } 2 \text { e } 3 \\
\text { anos, junto com um } \\
\text { de seus pais }\end{array}$ & $\begin{array}{l}\text { Linha de base múltipla } \\
\text { entre participantes }\end{array}$ & $\begin{array}{l}\text { RAC: ver Whalen e } \\
\text { Schreibman (2003) }\end{array}$ & $\begin{array}{l}\text { Treino de pais. Ensino RAC: TTD e } \\
\text { TRP; Treino de contato visual; } \\
\text { MES; DF e DV. SG } 3 \text { meses após } \\
\text { treinos. }\end{array}$ & $\begin{array}{l}\text { SD: BQ preferidos SRef: Con- } \\
\text { sequências naturais e Tangí- } \\
\text { veis }\end{array}$ & Sim & $\begin{array}{l}\text { Aquisição: Positiva } \\
\text { com emergência de } \\
\text { IAC Generalização: } \\
\text { Negativa para RAC }\end{array}$ \\
\hline
\end{tabular}




\begin{tabular}{|c|c|c|c|c|c|c|c|}
\hline Autores & $\begin{array}{c}\text { Perfil dos } \\
\text { participantes }\end{array}$ & $\begin{array}{l}\text { Delineamento } \\
\text { experimental }\end{array}$ & $\begin{array}{l}\text { Componente ACl } \\
\text { Classes de Resp. }\end{array}$ & Intervenção & Estímulos & $\begin{array}{l}\text { Partic. de } \\
\text { familiares }\end{array}$ & $\begin{array}{l}\text { Aquisição e } \\
\text { Generalização }\end{array}$ \\
\hline $\begin{array}{l}\text { MacDuff et al., } \\
\text { (2007) }\end{array}$ & $\begin{array}{l}3 \text { crianças com } \\
\text { autismo, entre } 3-5 \\
\text { anos. Presença de } \\
\text { A/M e IV } \\
3 \text { criancas com }\end{array}$ & $\begin{array}{l}\text { Linha de base múltipla } \\
\text { entre participantes }\end{array}$ & $\begin{array}{l}\text { IAC: Ativar um gravador, A } \\
\text { para SD, TO e repetir script }\end{array}$ & $\begin{array}{l}\text { Uso de script auditivo; Retirada } \\
\text { gradual da DF e do gravador; } \\
\text { Economia de fichas; SG }\end{array}$ & $\begin{array}{l}\text { SD: Objetos, BQ preferidos } \\
\text { e fotografias. SRef: fichas e } \\
\text { comestíveis, depois retirada } \\
\text { destes últimos }\end{array}$ & Não & $\begin{array}{l}\text { Aquisição e Gene- } \\
\text { ralização: } \\
\text { Positivas para IAC }\end{array}$ \\
\hline $\begin{array}{l}\text { Naoi et al., } \\
\text { (2008) }\end{array}$ & $\begin{array}{l}\text { autismo, entre 4-7 } \\
\text { anos. Presença de } \\
\text { IV, imitação motora } \\
\text { e mando }\end{array}$ & $\begin{array}{l}\text { Linha de base múltipla } \\
\text { entre participantes }\end{array}$ & IAC: TO, A, C, AF & $\begin{array}{l}\text { TTD; modelação; SG imediatamen- } \\
\text { te após treino e } 2 \text { meses depois }\end{array}$ & $\begin{array}{l}\text { SD: BQ preferidos. } \\
\text { SRef: Social }\end{array}$ & Não & $\begin{array}{l}\text { Aquisição e Gene- } \\
\text { ralização: } \\
\text { Positivas para IAC }\end{array}$ \\
\hline $\begin{array}{l}\text { Taylor e Hoch } \\
\text { (2008) }\end{array}$ & $\begin{array}{l}3 \text { crianças com } \\
\text { autismo, entre 3-8 } \\
\text { anos. Presença de } \\
\text { mando e tato }\end{array}$ & $\begin{array}{l}\text { Linha de base múltipla } \\
\text { entre participantes }\end{array}$ & $\begin{array}{l}\text { RAC: OA; C; TO } \\
\text { IAC: C; TO }\end{array}$ & $\begin{array}{l}\text { TTD para RAC e IAC; Hierarquia de } \\
\text { DG, DF e DV. Reforçamento positi- } \\
\text { vo e negativo; SG após treino em } \\
\text { locais diferentes }\end{array}$ & $\begin{array}{l}\text { SD: BQ não familiares e famili- } \\
\text { ares; SRef: Social }\end{array}$ & Não & $\begin{array}{l}\text { Aquisição: Positiva } \\
\text { Generalização: } \\
\text { Negativa para IAC }\end{array}$ \\
\hline $\begin{array}{l}\text { Isaksen e Holth } \\
\text { (2009) }\end{array}$ & $\begin{array}{l}4 \text { crianças com } \\
\text { autismo, de 3-5 } \\
\text { anos. Presença de } \\
\text { tato, mando, IV, } \\
\text { imitação motora }\end{array}$ & $\begin{array}{l}\text { Linha de base múltipla } \\
\text { entre participantes }\end{array}$ & $\begin{array}{l}\text { RAC: OA; TO } \\
\text { IAC: TO }\end{array}$ & $\begin{array}{l}\text { Treino de RAC e IAC ver Whalen e } \\
\text { Schreibman (2003); Treino de } \\
\text { DMO; BTT para RAC e IAC; Treino } \\
\text { de pais; SG } 1 \text { mês após o treino }\end{array}$ & $\begin{array}{l}\text { SD: BQ novos, não familiares à } \\
\text { criança. } \\
\text { SRef: consequências naturais e } \\
\text { sociais }\end{array}$ & Sim & $\begin{array}{l}\text { Aquisição e Gene- } \\
\text { ralização: } \\
\text { Positivas para RAC } \\
\text { e IAC }\end{array}$ \\
\hline $\begin{array}{l}\text { Klein et al., } \\
\text { (2009) }\end{array}$ & $\begin{array}{l}3 \text { crianças com } \\
\text { autismo, entre 4-6 } \\
\text { anos }\end{array}$ & $\begin{array}{l}\text { Linha de base múltipla } \\
\text { entre participantes }\end{array}$ & RAC: DMO & $\begin{array}{l}\text { TTD; Treino com ativação do BQ: } \\
\text { atrasada, contingente e intermiten- } \\
\text { te; RT; SG }\end{array}$ & $\begin{array}{l}\text { SD: BQ de ativação mecânica; } \\
\text { Interação social com } E \text {. } \\
\text { SRef: ativação de } B Q \text {, conse- } \\
\text { quências naturais e interações } \\
\text { sociais. }\end{array}$ & Não & $\begin{array}{l}\text { Aquisição e Gene- } \\
\text { ralização: } \\
\text { Positivas para RAC }\end{array}$ \\
\hline $\begin{array}{l}\text { Kasari, Guls- } \\
\text { rud, Wong, } \\
\text { Kwon e Locke } \\
\text { (2010) }\end{array}$ & $\begin{array}{l}38 \text { crianças com } \\
\text { autismo, entre } 1 \text { ano } \\
\text { e meio e } 3 \text { anos }\end{array}$ & $\begin{array}{l}\text { Delineamento de } \\
\text { grupo }\end{array}$ & $\begin{array}{l}\text { RAC e IAC: Ver Kasari et. } \\
\text { al (2006) }\end{array}$ & $\begin{array}{l}\text { Treino de pais. Ver Kasari et. al } \\
\text { (2006) para ensino de RAC e IAC }\end{array}$ & Ver Kasari et. al (2006) & Sim & $\begin{array}{l}\text { Aquisição: Positivas } \\
\text { Generalização: } \\
\text { Positiva para RAC e } \\
\text { inconsistente para } \\
\text { IAC } \\
\text { Aquisição: }\end{array}$ \\
\hline Jones (2009) & $\begin{array}{l}2 \text { crianças com } \\
\text { autismo, de } 3 \text { e } 4 \\
\text { anos, com repertório } \\
\text { estabelecido de } \\
\text { RAC }\end{array}$ & $\begin{array}{l}\text { Linha de base múltipla } \\
\text { entre respostas }\end{array}$ & IAC: TO, A, C & $\begin{array}{l}\text { Estudo 1: Ensino de IAC: TTD para } \\
\text { P1 e TI para P2; Sequência de } \\
\text { treino: 1. Ensino de TO; } 2 \text {. TO e A; } \\
\text { 3. TO, A e C. Uso scripts. Estudo 2: } \\
\text { Ensino de IAC: TI na rotina para } \\
\text { P1; Treino de pares e professores. } \\
\text { Hierarquia e DF }\end{array}$ & $\begin{array}{l}\text { ESTUDO 1: SD: BQ de ativa- } \\
\text { ção, Jogos preferidos. } \\
\text { ESTUDO 2: SD: Jogos e } \\
\text { atividades de lazer (montar } \\
\text { quebra-cabeça) e de rotina } \\
\text { (almoço) } \\
\text { SRef: sociais e consequências } \\
\text { naturais de ambos estudos }\end{array}$ & Não & $\begin{array}{l}\text { ESTUDO 1: Positi- } \\
\text { vo para de IAC. } \\
\text { ESTUDO 2: Positi- } \\
\text { va para duas das } \\
\text { três situações de } \\
\text { rotina. } \\
\text { Generalizaçãa: } \\
\text { Inconsistente para } \\
\text { IAC em novas } \\
\text { rotinas }\end{array}$ \\
\hline
\end{tabular}




\begin{tabular}{|c|c|c|c|c|c|c|c|}
\hline Autores & $\begin{array}{c}\text { Perfil dos } \\
\text { participantes }\end{array}$ & $\begin{array}{l}\text { Delineamento } \\
\text { experimental }\end{array}$ & $\begin{array}{l}\text { Componente ACl } \\
\text { Classes de Resp. }\end{array}$ & Intervenção & Estímulos & $\begin{array}{l}\text { Partic. de } \\
\text { familiares }\end{array}$ & $\begin{array}{l}\text { Aquisição e } \\
\text { Generalização }\end{array}$ \\
\hline $\begin{array}{l}\text { Kaale, Smith e } \\
\text { Sponheim } \\
(2012)\end{array}$ & $\begin{array}{l}61 \text { crianças com } \\
\text { autismo, entre } 2-5 \\
\text { anos }\end{array}$ & $\begin{array}{l}\text { Delineamento de } \\
\text { grupo }\end{array}$ & $\begin{array}{l}\text { IAC: Ver Kasari et. al } \\
(2006)\end{array}$ & $\begin{array}{l}\text { GEXP: Treino de IAC: TTD, TRP e } \\
\text { currículo escolar; Treino de profes- } \\
\text { sores para aplicação do procedi- } \\
\text { mento; } \\
\text { Hierarquia DF; SG após treino. } \\
\text { GCONT Currículo escolar. }\end{array}$ & $\begin{array}{l}\text { SD: BQ e objetos. SRef: con- } \\
\text { sequências sociais, naturais, } \\
\text { acesso ao SD. }\end{array}$ & Não & $\begin{array}{l}\text { Aquisição: Positiva } \\
\text { Generalização: } \\
\text { Inconsistente para } \\
\text { IAC }\end{array}$ \\
\hline $\begin{array}{l}\text { Pollard, Betz e } \\
\text { Higbee (2012) }\end{array}$ & $\begin{array}{l}3 \text { crianças com } \\
\text { autismo, entre } 4 \text { - } 7 \\
\text { anos, com repertó- } \\
\text { rios de linguagem e } \\
\text { leitura }\end{array}$ & $\begin{array}{l}\text { Sujeito único, Linha } \\
\text { de base múltipla entre } \\
\text { participantes }\end{array}$ & $\begin{array}{l}\text { IAC: Olhar para o SD, A, } \\
\text { ler script em voz alta e TO }\end{array}$ & $\begin{array}{l}\text { Múltiplos scripts (dicas textuais) } \\
\text { para ensino de IAC; Retirada } \\
\text { gradual do script; Variação dos } \\
\text { ambientes treinados; DF e DV com } \\
\text { e sem atraso; Modelação; Proce- } \\
\text { dimento de correção; SG } 6 \text { sema- } \\
\text { nas após treino }\end{array}$ & $\begin{array}{l}\text { SD: Objetos e BQ preferidos } \\
\text { SRef: sociais }\end{array}$ & Não & $\begin{array}{l}\text { Aquisição e Gene- } \\
\text { ralização: } \\
\text { Positivas para IAC }\end{array}$ \\
\hline $\begin{array}{l}\text { Krstovska- } \\
\text { Guerrero e } \\
\text { Jones (2013) }\end{array}$ & $\begin{array}{l}3 \text { crianças com } \\
\text { autismo, entre } 2 \text { e } 4 \\
\text { anos, apresentando } \\
\text { seguimento de } \\
\text { instruções }\end{array}$ & $\begin{array}{l}\text { Linha de base múltipla } \\
\text { entre as respostas }\end{array}$ & RAC: OA, S, TO & $\begin{array}{l}\text { Ensino de RAC: TTD 1. S; 2. OA e } \\
\text { S; 3. TO e S. Hierarquia DF (cóce- } \\
\text { gas para sorrir), DV com atraso; } \\
\text { MES; SG com pais imediatamente } \\
\text { após treino }\end{array}$ & $\begin{array}{l}\text { SD: BQ e atividades preferidos } \\
\text { SRef: ER com BQ preferidos/ } \\
\text { comestíveis e TRC de intera- } \\
\text { ção social. }\end{array}$ & Sim & $\begin{array}{l}\text { Aquisição e Gene- } \\
\text { ralização: } \\
\text { Positivas para RAC }\end{array}$ \\
\hline $\begin{array}{l}\text { Warreyn e } \\
\text { Roeyers (2013) }\end{array}$ & $\begin{array}{l}36 \text { cças com autis- } \\
\text { mo, entre } 3-7 \text { anos, } \\
\text { apresentando } \\
\text { seguimento de } \\
\text { instruções simples }\end{array}$ & $\begin{array}{l}\text { Delineamento de } \\
\text { grupo }\end{array}$ & $\begin{array}{l}\text { RAC: OA, DMO } \\
\text { IAC: TO }\end{array}$ & $\begin{array}{l}\text { GEXP: Treino de AC, Imitação e } \\
\text { currículo escolar; Treino de RAC e } \\
\text { IAC através de TRP. GCONT: } \\
\text { Currículo escolar. }\end{array}$ & $\begin{array}{l}\text { SD: BQ e objetos preferidos } \\
\text { SRef: interações sociais e } \\
\text { consequências naturais. }\end{array}$ & Não & $\begin{array}{l}\text { Aquisição: Positivas } \\
\text { para RAC e IAC } \\
\text { Generalização: Não } \\
\text { houve SG }\end{array}$ \\
\hline Wong (2013) & $\begin{array}{l}33 \text { crianças com } \\
\text { autismo, entre 3- } 6 \\
\text { anos }\end{array}$ & $\begin{array}{l}\text { Delineamento de } \\
\text { grupo }\end{array}$ & $\begin{array}{l}\text { RAC: AO, DMO } \\
\text { IAC: TO, A, M }\end{array}$ & $\begin{array}{l}\text { Grupo AC/BS; Grupo BS/AC; } \\
\text { Grupo controle. Treino de AC e BS } \\
\text { ver Kasari et. al. (2006). }\end{array}$ & ver Kasari et. al. (2006) & Não & $\begin{array}{l}\text { Aquisição: Positivas } \\
\text { RAC e IAC Genera- } \\
\text { lização: Positiva } \\
\text { RAC e Incons. para } \\
\text { IAC }\end{array}$ \\
\hline
\end{tabular}

Legenda: A: Apontar para o estímulo; AC: Atenção Conjunta; AF: Abordar fisicamente o experimentador; A/M: Apontar como mando; BQ: Brinquedos; BS: Brincadeira simbólica; BTT: Brincadeira com troca de turno; C: Comentar sobre o estímulo; CN: Chamar a criança pelo nome; CV: Comportamento verbal; DF: Dica física; DG: Dica gestual; DMO: Discriminar a mudança no foco do olhar do experimentador; DV: Dica verbal; E: Experimentador; EO: Entregar o objeto; ER: Esquema de reforçamento; GC: Girar a cabeça; GEXP: Grupo experimental; GCONT: Grupo Controle; IV: Imitação vocal; M: Mostrar o objeto para adulto; MES: Múltiplos exemplares de estímulos; MF: Mudar o foco do olhar em direção ao objeto; OA: Olhar para um objeto apontado pelo experimentador; P: Participante; RT: Randomização entre tarefas de manutenção e de aquisição; S: Sorrir; SD: Estímulos discriminativos; SG: Sonda de generalização; SRef: Estímulos reforçadores; TI: Treino incidental; TO: Trocar olhar entre o objeto e o experimentador; TRC: Taxa de reforçamento contínuo; TRP: Treino de Resposta Pivotal; TTD: Treino de tentativa discreta. 
Nos estudos de Kasari et al. (2006, 2010) e Isaksen e Holth (2009) a classe de resposta treinada na RAC, de olhar para um estímulo apontado por E, ocorreu conforme o protocolo de MacDonald et al. (2006), o qual propõe que o estímulo esteja em duas distâncias diferentes em relação à criança e em posições diversas. Topografias como alternar o olhar entre o objeto e E. (Isaksen \& Holth, 2009; Krstovska-Guerreiro \& Jones 2013; Taylor \& Hoch, 2008), comentar (Taylor \& Hoch, 2008) e sorrir (Krstovska-Guerreiro \& Jones, 2013) apareceram nos treinos como classes mais avançadas coordenadas com 'olhar para um estímulo apontado por E'.

B2. Classes de Respostas de IAC: todos os estudos que buscavam ensinar a IAC treinaram-na a partir da forma mais simples de 'alternar o olhar entre o objeto e E' (Gulsrud et al., 2007; Isaksen \& Holth, 2009; Jones, 2009; Jones et al., 2006; Kaale et al., 2012; Kasari et al., 2006, 2010; MacDuff et al., 2007; Naoi et al., 2008; Pollard et al., 2012; Taylor \& Hoch, 2008; Warreyen \& Royers, 2013; Whalen \& Schreibman, 2003; Wong 2013). Destes estudos, excetuandose três deles (Isaksen \& Holth, 2009; Taylor \& Hoch, 2008; Warreyen \& Royers, 2013), os demais ensinaram a topografia de apontar para o estímulo conjugada com a forma anterior. A resposta de mostrar o estímulo apareceu em cinco estudos (Gulsrud et al., 2007; Kaale et al., 2012; Kasari et al., 2006, 2010; Wong 2013) e a de entregar o estímulo em três (Kaale et al., 2012; Kasari et al., 2006, 2010). A resposta de comentar sobre o estímulo foi treinada em três estudos (Jones, 2009; Naoi et al., 2008; Taylor \& Hoch, 2008), sendo que dois utilizaram dicas textuais (scripts) para auxiliar a criança a vocalizar sobre o estímulo (MacDuff et al., 2007; Pollard et al., 2012).

C. Características da intervenção: a maioria dos estudos que ensinou RAC o fez através de um treino de tentativa discreta, com as exceções de Warreyn e Royers (2013 - treino de resposta pivotal), Rocha et al. (2007 - tentativa discreta e resposta pivotal) e (Isaksen \& Holth, 2009 - condição de brincadeira de troca de turno com E). No procedimento de resposta pivotal (ensino baseado em oportunidades de aprendizagem que ocorrem no ambiente natural das crianças, focado em respostas consideradas pivotais para o desenvolvimento - ex. responsividade para múltiplos estímulos, motivação) eram arranjados no ambiente estímulos com propriedades discriminativas realçadas, aumentando a probabilidade de uma resposta discriminada da criança. No treino na condição de brincadeira de troca de turno com E, o experimentador e a criança se engajavam na construção de blocos e montagem de quebra cabeças. Era considerada uma resposta correta de RAC quando a criança pegava o bloco ou a peça de quebra cabeça e começava a construção, após as respostas do experimentador de olhar e apontar para a peça. Para o ensino de RAC com tentativas discretas (ensino baseado em apresentações estruturadas de oportunidades de aprendizagem), enquanto a criança brincava com um estímulo, o experimentador apontava, olhava e/ou comentava sobre um novo brinquedo. A criança deveria alternar o olhar entre o item e o experimentador e interagir com o novo brinquedo. Na ausência de RAC ou IAC no treino inicial com tentativas discretas, 15 estudos introduziram uma hierarquia de dicas físicas, gestuais e/ou verbais para evocar a resposta correta (Gulsrud et al., 2007; Isaksen \& Holth, 2009; Jones, 2009; Jones et al., 2006; Kaale et al., 2012; Kasari et al., 2006, 2010; Krstovska-Guerrero \& Jones, 2013; MacDuff et al., 2007; Martins \& Harris, 2006; Pollard et al., 2012; Rocha et al., 2007; Taylor \& Hoch, 2008; Whalen \& Schreibman, 2003; Wong, 2013). Um estudo (Klein et al., 2009) usou a ativação de um brinquedo como uma dica inerente ao próprio estímulo.

O ensino de IAC ocorreu através de treino de resposta pivotal (Isaksen \& Holth, 2009; Jones et al., 2006; Kaale et al., 2012; Warreyn \& Royers, 2013; Whalen \& Schreibman, 2003; Wong 2013), treino incidental em que eram envolvidas atividades já conhecidas, de interesse ou de rotina da criança (Gulsrud et al., 2007; Jones, 2009; Kasari et al., 2006, 2010), tentativas discretas (Jones, 2009; Kaale et al., 2012; MacDuff et al., 2007; Naoi et al., 2008; Pollard et al., 2012; Taylor \& Hoch, 2008;) e modelação (Jones et al., 2006; Naoi et al., 2008). O treino incidental se assemelha ao de resposta pivotal: a oportunidade de iniciar uma tentativa de IAC é controlada pela atenção da criança; são arranjados no ambiente estímulos de propriedades discriminativas realçadas, aumentando a probabilidade de respostas de iniciação da criança e interações com o Experimentador.

Todos os estudos analisados utilizaram reforçamento positivo para ensinar os componentes de AC. 
Um estudo empregou também punição negativa (Whalen \& Schreibman, 2003) com a retirada do brinquedo caso a resposta de IAC fosse incorreta, e um estudo (Taylor \& Hoch, 2008) procurou instalar IAC através de contingências de reforçamento negativo usando estímulos discriminativos com propriedades aversivas.

D. Estímulos discriminativos e reforçadores: na seleção de estímulos discriminativos, dez estudos usaram brinquedos, jogos ou atividades preferidas e de escolha da criança (Jones, 2009; Jones et al., 2006; Krstovska-Guerrero \& Jones, 2013; MacDuff et al., 2007; Martins \& Harris, 2006; Naoi et al., 2008; Pollard et al., 2012; Rocha et al., 2007; Taylor \& Hoch, 2008; Warreyen \& Royers, 2013), oito utilizaram brinquedos de ativação (Gulsrud et al., 2007; Jones, 2009; Jones et al., 2006; Kaale et al., 2012; Kasari et al., 2006, 2010; Klein et al., 2009; Wong 2013) e três usaram interações físicas com E (Kasari et al., 2006, 2010; Klein et al., 2009). Dois estudos usaram painéis com figuras (Jones et al., 2006; Whalen \& Schreibman, 2003;), e outros dois empregaram brinquedos novos, não conhecidos pelos participantes (Isaksen \& Holth, 2009; Taylor \& Hoch, 2008). MacDuff et al. (2007) utilizaram fotografias.

Quanto aos reforçadores, 14 estudos usaram consequências naturais, ou seja, consequências relacionadas ao próprio estímulo discriminativo apresentado (Gulsrud et al., 2007; Isaksen \& Holth, 2009; Jones, 2009; Jones et al., 2006; Kaale et al., 2012; Kasari et al., 2006, 2010; Klein et al., 2009; KrstovskaGuerrero \& Jones, 2013; Martins \& Harris, 2006; Rocha et al., 2007; Warreyen \& Royers, 2013; Whalen \& Schreibman, 2003; Wong 2013), seis usaram consequências tangíveis como manipulação de brinquedos ou comestíveis (Jones et al., 2006; KrstovskaGuerrero \& Jones, 2013; MacDuff et al., 2007; Martins \& Harris, 2006; Rocha et al., 2007; Whalen \& Schreibman, 2003), e onze introduziram consequências sociais junto com uma das mencionadas antes (Gulsrud et al., 2007; Isaksen \& Holth, 2009; Jones, 2009; Jones et al., 2006; Kaale et al., 2012; Kasari et al., 2006, 2010; Klein et al., 2009; KrstovskaGuerrero \& Jones, 2013; Martins \& Harris, 2006; Warreyn \& Royers, 2013; Wong, 2013). Três estudos usaram consequências exclusivamente sociais (Naoi et al., 2008; Pollard et al., 2012; Taylor \& Hoch, 2008) e um usou economia de fichas (MacDuff et al., 2007).
E. Participação de familiares no procedimento: Sete estudos introduziram pessoas familiares à criança no procedimento, tanto nos testes de generalização dos repertórios após o treino (Gulsrud et al., 2007; Kasari et al., 2006; Krstovska-Guerrero \& Jones, 2013) quanto na modalidade de treino parental (Isaksen \& Holth, 2009; Jones et al., 2006; Kasari et al., 2010; Rocha et al., 2007), com resultados positivos na generalização dos repertórios treinados na maioria dos estudos.

F. Aquisição e generalização: Todos os artigos analisados foram bem sucedidos em estabelecer RAC e/ou IAC, mas o resultado de generalização e manutenção do repertório de IAC variou entre estudos. O sucesso da generalização e manutenção da IAC parece ter sido afetado positivamente pelo ensino com treino de resposta pivotal ou incidental, à familiaridade e/ou preferência dos estímulos antecedentes, às consequências sociais oferecidas no treino e/ou à inclusão de familiares no treino (Gulsrud et al., 2007; Isaksen \& Holth, 2009; Jones et al., 2006; Kasari et al., 2006; Naoi et al., 2008; Pollard et al., 2012).

\section{DISCUSSÃO}

Considerados conjuntamente, os 18 estudos analisados mostraram que a AC pode ser ensinada para pessoas com autismo por meio de intervenções comportamentais, mas que a RAC, replicando os dados da literatura sobre AC, é mais facilmente instalada do que a IAC (MacDonald et al., 2006; Meindl \& Cannella-Malone, 2011; White et al., 2011). Uma das explicações para as dificuldades da aquisição de IAC parece estar na exigência de um pré-requisito de "motivação social" a qual é estabelecida quando as consequências mediadas pelos adultos já adquiriram função reforçadora (Isaksen \& Holth, 2009). Os resultados dos estudos mostraram uma aquisição consistente de RAC mantida através de contingências de reforçamento generalizado, tangível e/ou exclusivamente social. No entanto, a aquisição da RAC não levou a emergência de IAC, o que oferece suporte para a sugestão de que esses repertórios são funcionalmente independentes (Dube et al., 2004). Além disso, no estudo de Martins e Harris (2006) a RAC foi mantida na ausência de reforçamento específico, sugerindo que esse repertório pode também ser independente (ao menos na etapa de aquisição) do operante verbal mando. 
Os resultados dos estudos também mostram que uma condição prévia que parece facilitar o desenvolvimento de IAC é o refinamento da topografia de resposta no treino de RAC, exigindo que a criança não só olhe para um item sendo apontado pelo adulto, mas também siga a mudança no foco do olhar do outro. Esse refinamento parece facilitar a discriminação, por parte da criança, do comportamento do adulto de atentar para estímulos, o que, por sua vez, contribui para estabelecer esse comportamento do adulto como estímulos condicionais discriminativos e/ou reforçadores (ver Dube et al., 2004; Holth, 2005).

Para o estabelecimento das respostas de IAC parece importante avaliar, em condições naturais, que estímulos antecedentes ou propriedades de estímulos podem funcionar como uma operação motivadora (OM), no sentido de aumentar a probabilidade da emissão dessas respostas no treino. Os dados têm indicado que as crianças olham mais para os estímulos conhecidos que consideram interessantes, o que sugere a natureza idiossincrática da OM para estabelecer oportunidades de IAC em função dos interesses restritos de crianças com autismo. Isso alerta sobre o risco de selecionar previamente, com base nos julgamentos do experimentador, quais estímulos poderão ter propriedades discriminativas realçadas e evocativas de IAC.

Outro aspecto que parece importante para o estabelecimento das respostas de IAC é o pareamento da presença de outra pessoa com uma variedade de estímulos reforçadores tangíveis, promovendo o aumento do valor reforçador da interação social e da probabilidade de estabelecer os comportamentos das outras pessoas como OM(s). Essa relação entre a efetividade com que o comportamento de outras pessoas funciona como estímulos condicionais discriminativos e/ou reforçadores e a aquisição de RAC e IAC deve ser investigada de forma mais detalhada em estudos futuros.

Dois estudos foram bem sucedidos em promover aquisição, generalização e manutenção do repertório de IAC através de dicas auditivas e textuais (MacDuff et al., 2007; Pollard et al., 2012) em crianças que já tinham repertórios verbais estabelecidos (ex. mando, tato $^{3}$, leitura). No estudo de MacDuff et al. (2007) todos os participantes aprenderam a IAC apontando para o gravador e repetindo a dica auditiva. Quando a dica foi retirada, as crianças não só continuaram a repetir a dica, mas também começaram a usar novas construções verbais (ex. tato com autoclítico ${ }^{4}$. No estudo de Pollard et al. (2012) as crianças eram ensinadas a emitir as respostas de apontar para o estímulo e ler em voz alta a dica textual, tendo como consequência comentários sobre o estímulo feitos pelo experimentador. Foi observado que na condição na qual o experimentador oferecia múltiplos exemplares de comentários após a emissão de IAC pela criança, houve um aumento de repertório verbal independente (sem dica) para dois dos três participantes, em comparação com a condição na qual os comentários eram os mesmos. Esses resultados mostram uma relação de facilitação mútua entre repertórios verbais básicos e IAC que precisa ser explorada em novos estudos.

Os resultados dos artigos analisados sugerem que as intervenções mediadas pelos pais ou professores são efetivas em estabelecer $\mathrm{AC}$, além de repertórios sociais importantes que são deficientes em crianças com autismo. Porém, alguns estudos que fizeram treino de pais/professores não conseguiram manter os repertórios nos testes de manutenção realizados meses após o treino (Jones, 2009; Kaale et al., 2012; Kasari et al., 2010; Rocha et al., 2007). A explicação para essa inconsistência pode estar relacionada às diferenças individuais entre os pais ao ensinarem as diferentes classes de respostas que constituem a RAC e a IAC. Observou-se que a maioria dos pais implementou com maior frequência e precisão as primeiras fases do treino (e.g segurar e mostrar um estímulo), do que as últimas (e.g apontar e alternar olhar entre objeto e a criança) (e.g. Rocha et al., 2007). Outro problema que pode ter levado a falhas na generalização é que os pais foram instruídos, em ambientes controlados, a oferecer oportunidades de RAC e IAC para a criança, mas não foram diretamente ensinados a como fazer isso em ambiente natural. Rocha et al. (2007) apontam que os pais apresentaram dificuldade em discriminar e oferecer todas as oportunidades para que as crianças apresentassem RAC e IAC e sugerem que o os treinos de pais sejam estendidos aos ambientes naturais.

No que diz respeito à generalização e manutenção dos repertórios, os estudos mostraram resultados inconsistentes. Na maioria dos estudos a intervenção em AC ocorreu em contexto estruturado, não natural e através de tentativas discretas, o que levou a um aprendizado eficiente na situação específica de ensino, 
mas sem generalização para contextos naturais. Os estudos que obtiveram maior sucesso foram os que introduziram uma condição de brincadeira no procedimento e treinaram os repertórios em diversas condições (Gulsrud et al., 2007; Jones, 2009; Jones et al., 2006; Kasari et al., 2006, 2010; Isaksen \& Holth, 2009; Pollard et al., 2012; Wong, 2013) ou aqueles que empregaram procedimentos de ensino naturalísticos (Martins \& Harris, 2006), incorporando variáveis motivacionais no ensino que podem ter contribuído para a generalização dos repertórios.

De maneira geral, os procedimentos mais eficazes para ensinar RAC e IAC utilizaram as estratégias analítico-comportamentais (e.g. hierarquia de dicas, variação de reforçamento, uso de múltiplos exemplares de estímulos, testes de preferências, modelação e randomização de tarefas) inseridas em treinos que envolviam a participação de adultos familiares em condições naturais do desenvolvimento da criança (Gulsrud et al., 2007; Isaksen \& Holth, 2009; Krstovska-Guerrero \& Jones, 2013). Pesquisas futuras devem avaliar os efeitos isolados e combinados dessas variáveis para o ensino, expansão e manutenção dos repertórios de AC.

Uma questão de grande relevância, considerandose a relação entre AC e desenvolvimento verbal sugerida pela literatura (Charman, 2003; White et al., 2011), mas que nenhum dos estudos analisados abordou diretamente, diz respeito à extensão dos efeitos da aquisição da RAC e ou IAC sobre a aprendizagem de operantes verbais básicos (e.g. mando, tato). Alguns estudos longitudinais, não incluídos nessa revisão (e.g. Kasari, Paparella, Freeman, \& Jahromi, 2008; Kasari, Gulsrud, Freeman, Paparella, \& Hellemann, 2012; Morales et al., 2000; Whalen et al. 2006), mostraram efeitos positivos da aquisição de AC no desenvolvimento da linguagem e de outras habilidades sociais. Esses resultados oferecem suporte à hipótese de que a habilidade de AC é uma cúspide comportamental (behavioral cusp - ver Rosales-Ruiz \& Baer, 1997) que permite o desenvolvimento de repertórios mais avançados (Greer \& Ross, 2008). Essa hipótese, aplicada ao contexto da relação entre $\mathrm{AC}$ e desenvolvimento verbal em crianças com autismo, deve levar a pesquisas futuras (básicas e/ou aplicadas) sobre as relações funcionais entre RAC, IAC e a aquisição de operantes verbais nessa população.

\section{REFERÊNCIAS}

American Psychiatric Association. (2013). Diagnostic and statistical manual of mental disorders $\left(5^{\text {th }}\right.$ ed.). Arlington, VA: American Psychiatric Publishing.

Charman, T. (2003). Why is joint attention a pivotal skill in autism? Philosophical transactions of the royal society biological sciences, 358, 315-324.

Dube, W. V., MacDonald, R. P. F., Mansfield, R. C., Holcomb, W. L., \& Ahearn, W. H. (2004). Toward a behavioral analysis of joint attention. The Behavior Analyst, 27, 197-207.

Greer, D. R., \& Ross, D. E. (2008). Verbal behavior analysis: Inducing and expanding new verbal capabilities in children with language delays. Boston: Pearson Education, Inc.

Gulsrud, A. C., Kasari, C., Freeman, S., \& Paparella, T. (2007). Children with autism's response to novel stimuli while participating in interventions targeting joint attention or symbolic play skills. Autism, 11, 535-546.

Holth, P. (2005). An operant analysis of joint attention skills. Journal of Early and Intensive Behavior Intervention, 2, 160-175.

Isaksen, J., \& Holth, P. (2009). An operant approach to teaching joint attention skills to children with autism. Behavioral Interventions, 24, 215-236.

Jones, E. A. (2009). Establishing response and stimulus classes for initiating joint attention in children with autism. Research in Autism Spectrum Disorders, 3, 375389.

Jones, E. A., Carr, E. G., \& Feeley, K. M. (2006). Multiple effects of joint attention intervention for children with autism. Behavior Modification, 30, 782-834.

Kaale, A., Smith, L., \& Sponheim, E. (2012). A randomized controlled trial of preschool-based joint attention intervention for children with autism. Journal of Child Psychology and Psychiatry, 53, 97-105.

Kasari, C., Freeman, S., \& Paparella, T. (2006). Joint attention and symbolic play in young children with autism: A randomized controlled intervention study. Journal of Child Psychology and Psychiatry, 47, 611-620.

Kasari, C., Gulsrud, A., Freeman, S., Paparella, T., \& Hellemann, G. (2012). Longitudinal follow up of children with ASD receiving targeted interventions on joint attention and play. Journal of the American Academy of Child and Adolescent Psychiatry, 51, 487-495.

Kasari, C., Gulsrud, A. C., Wong, C., Kwon, S., \& Locke, J. (2010). Randomized controlled caregiver mediated joint engagement intervention for toddlers with autism. 
Journal of Autism and Developmental Disorder, 40, 1.045-1.056.

Kasari, C., Paparella, T., Freeman, S., \& Jahromi, L. (2008). Language outcome in autism: Randomized comparison of joint attention and play interventions. Journal of Consulting and Clinical Psychology, 76, 125-137.

Klein, J. L., McDonald, R. P. F., Villancout, G., Ahearn, W., \& Dube, W. V. (2009). Teaching discrimination of adult gaze direction to children with autism. Research in Autism Spectrum Disorders, 3, 42-49.

Krstovska-Guerrero, I., \& Jones, E. A. (2013). Joint attention in autism: Teaching smiling coordinated with gaze to respond to joint attention bids. Research in Autism Spectrum Disorders, 7, 93-108.

Langthorne, P., \& McGill, P. (2009). A tutorial on the concept of the motivating operation and its importance to application. Behavior Analysis in Practice, 2, 22-31.

MacDuff, J. L., Ledo, R., McClannahan, L. E., \& Krantz, P. J. (2007). Using scripts and script-fading procedures to promote bids for joint attention by young children with autism. Research in Autism Spectrum Disorders, 1, 281290.

Martins, M. P., \& Harris, S. L., (2006). Teaching children with autism to respond to joint attention initiations. Child \& Family Behavior Therapy, 28, 51-68.

McDonald, R, Anderson, J, Dube, W. V., Geckeler, A., Green, G., Holcomb, W., Mansfiel, R., \& Sanchez, J. (2006). Behavioral assessment of joint attention: A methodological report. Research in Developmental Disabilities, 27, 138-150.

Meindl, J. N., \& Cannella-Malone, H. I. (2011). Initiating and responding to joint attention bids in children with autism: A review of the literature. Research in Developmental Disabilities, 32, 1441-1454.

Morales, M., Mundy, P., Delgado, C., Yale, M., Messinger, D., Neal, R., \& Schwartz, H. (2000). Responding to joint attention across the 6-through 24-month age period and early language acquisition. Journal of Applied Developmental Psychology, 21, 283-298.

Mundy, P., Block, J., Delgado, C., Pomares, Y., Vaughan van Hecke, A., \& Venezia Parlade, M. (2007). Individual differences and the development of joint attention in infancy. Child Development, 78, 938-954.

Mundy, P., Delgado, C., Block, J., Venezia, M., Hogan, A., \& Seibert, J. (2003) A Manual for the abridged early social communication scales (ESCS). Cora Gables, FL: University of Miami, Department of Psychology. Recuperado dia 05/08/2012 de http://www.psy.miami.edu/fa culty/pmundy/main.phtml
Mundy, P., \& Newell, L. (2007). Attention, joint attention, and social cognition. Current directions in psychological science, 16, 269-274.

Naoi, N., Tsuchiya R., Yamamoto, J., \& Nakamura, K. (2008). Functional training for initiating joint attention in children with autism. Research in Developmental Disabilities, 29, 595-609.

Pollard, J. S., Betz, A. M., \& Higbee, T. S. (2012). Script fading to promote unscripted bids for joint attention in children with autism. Journal of Applied Behavior Analysis, 45, 387-393.

Rocha, M. L., Schreibman, L., \& Stahmer, A. C. (2007). Effectiveness of training parents to teach joint attention in children with autism. Journal of Early Intervention, $29,154-172$.

Rosales-Ruiz, J., \& Baer, D. M. (1997). Behavioral cusps: A developmental and pragmatic concept for behavior analysis. Journal of Applied Behavior Analysis, 30, 533544.

Skinner, B. F. (1992). Verbal Behavior. Acton, Massachusetts: Copley Publishing Group (Publicado originalmente em 1957).

Tager-Flusberg, H., Paul, R., \& Lord, C. (2005). Language and communication in autism. In F. Volkmar, R. Paul, A. Klin, \& D. Cohen (Eds.), Handbook of autism and pervasive developmental disorders (pp. 335-364). New Jersey: John Wiley \& Sons Inc.

Taylor, B. A., \& Hoch, H. (2008). Teaching children with autism to respond to and initiate bids for joint attention. Journal of Applied Behavior Analysis, 41, 377-391.

Tsatsanis, K. (2005). Neuropsychological characteristics in autism and related conditions. In F. Volkmar, R. Paul, A. Klin, \& D. Cohen (Eds.), Handbook of autism and pervasive developmental disorders (pp. 365-381). New Jersey: John Wiley \& Sons Inc.

Warreyn, P., \& Roeyers, H. (2013). See what I see, do as I do: Promoting joint attention and imitation in preschoolers with autism spectrum disorder. Autism. Advance online publication. doi: 10.1177/1362361313493834

Whalen, C., \& Schreibman, L. (2003). Joint attention training for children with autism using behavior modification procedures. Journal of Child Psychology and Psychiatry, $44,456-468$.

Whalen, C., Schreibman, L., \& Ingersoll, B. (2006). The collateral effects of joint attention training on social initiations, positive affects, imitation, and spontaneous speech for young children with autism. Journal of Autism and Developmental Disorders, 36, 655-664. 
White, P. J., O'Reilly, M., Streusand, W., Levine, A., Sigafoss, J., Lancioni, G., Fragale, C., Pierce, N., \& Aguilar, J. (2011). Best practices for teaching joint attention: A systematic review of the intervention literature. Research in Autism Spectrum Disorders, 5, 1.283-1.295.

Recebido em: 10/12/2013 Última revisão em: 10/04/2014 Aceito em: 17/04/2014

Wong, C. S. (2013). A play and joint attention intervention for teachers of young children with autism: a randomized controlled pilot study. Autism, 17, 340-357.

\section{Notas:}

1 O mando é um operante verbal controlado por variáveis motivacionais (e.g. privação, punição) e mantido por reforçamento específico - Por exemplo, se uma pessoa me diz o seu telefone, e eu não tenho uma caneta para anotar o número, posso emitir o mando "Me empresta a sua caneta", que será reforçado pela entrega da caneta.

2 MO se refere a "um evento ou estímulo que momentaneamente altera (a) o valor das consequências que atuam como reforçamento ou punição, e (b) a probabilidade dos comportamentos que têm sido previamente associados a tais consequências" (Langthorne \& McGill, 2009, p. 22)

3 O tato é um operante verbal controlado por estímulos antecedentes não verbais e mantido por reforçamento generalizado (Skinner, 1957/1992) - Por exemplo, diante de um pássaro, um estudante de ornitologia diz "Serinus canaria", e o seu professor reforça esta resposta dizendo "Muito bem".

4 O autoclítico é um operante verbal de segunda ordem: comportamento verbal sob controle de outro comportamento verbal do falante, que pode ser caracterizado principalmente pelos seus efeitos sobre o ouvinte ao descrever, qualificar, quantificar ou relacionar para este as relações de controle da emissão do comportamento verbal de primeira ordem (Skinner, 1957/1992) - Por exemplo, duas pessoas estão buscando um endereço, e diante de uma casa uma delas diz "É esta casa, tenho certeza". 